\title{
Progressive Agriculture
}

\section{Effect of salinity on germination and early seedling growth of maize}

\author{
R Ahmed ${ }^{1}$, MHK Howlader ${ }^{1}$, A Shila ${ }^{1}$, MA Haque ${ }^{2 *}$ \\ ${ }^{1}$ Department of Agricultural Botany and ${ }^{2}$ Department of Soil Science, Patuakhali Science and Technology \\ University, Dumki, Patuakhali 8602, Bangladesh
}

\begin{abstract}
An incubation experiment was conducted at the Department of Soil Science, Patuakhali Science and Technology University during Rabi season 2015 to investigate the effects of different levels of salinity on germination and early seedling growth of maize. There were ten treatments consisting of different concentrations of salinity viz. $0,20,40$, $80,120,160,200,240,280$ and $320 \mathrm{mM} \mathrm{NaCl}$. The germination experiment was conducted in petri dish lined with a layer of cotton consisting ten $\mathrm{ml}$ of each test solution. Germination percentage gradually decreased with the increase of concentration of salt. Up to $80 \mathrm{mM}$ concentration was found safe for maize seed germination. Salinity caused delay in germination. Highest seedling height was found at $40 \mathrm{mM} \mathrm{NaCl}$ concentration $(21.51 \mathrm{~cm})$ and root length $(23.61 \mathrm{~cm}$ ) was found in $20 \mathrm{mM} \mathrm{NaCl}$ concentration. In $320 \mathrm{mM} \mathrm{NaCl}$ concentration roots were abnormal, deformed and twisted. The 0 to $80 \mathrm{mM} \mathrm{NaCl}$ concentration gave statistically similar shoot fresh weight. Compared to control treatment $20 \mathrm{mM} \mathrm{NaCl}$ concentration gave $0.55 \%$ higher fresh weight and at $80 \mathrm{mM} \mathrm{NaCl}$ concentration shoot fresh weight reduced by only $6.9 \%$. The $80 \mathrm{mM} \mathrm{NaCl}$ concentration was found acceptable for germination and early seedling growth of maize.
\end{abstract}

Key words: Coastal Bangladesh, sodium chloride, speed of germination, salinity, vigor index

Progressive Agriculturists. All rights reserve

*Corresponding Author: masadulh@yahoo.com

\section{Introduction}

Salinity is a major environmental constraint to crop productivity throughout the arid and semi-arid regions of the world (Foolad and Lin, 1997). In Bangladesh, there are approximately 2.85 million ha of coastal land of which about one million ha are seriously affected by varying degrees of salinity (SRDI, 2010). About 30$50 \%$ of net cropped areas remains fallow in rabi and Kharif-1 seasons, mainly due to soil and water (irrigation) salinity. The salinity increases in dry months showing a peak in March-April and decreases in wet months with the minimum in July-August.

Annual precipitation does not exceed evapotranspiration, soluble salts tend to accumulate and build-up in the soil of coastal regions, instead of being leached, and can reach the levels inhibitory to plant growth and development (Roychoudhury and Chakraborty, 2013). The prevailing salinity intrusion due to climate change is severely affecting the crop productivity in the saline regions of Bangladesh (Haque et al., 2014). The situation calls an urgent need to improve crop productivity. Introduction of new crops and/or crop varieties in the fallow lands of the coastal regions might be the scholastic technique for improvement of system productivity. Maize is one of the most important food grains in the world as well as in developing countries like Bangladesh.

The food produced in Bangladesh is not adequate to meet domestic requirements. Seed germination is a 
major factor limiting the establishment of plants under saline conditions (Carpıc1 et al., 2009). Salinity may cause significant reductions in the rate and percentage of germination, which in turn may lead to uneven stand establishment and reduced crop yields (Foolad et al., 1999).

Salt tolerance at germination stage is important factor, where soil salinity is mostly dominated at surface layer. High concentration of salt has detrimental effects on germination of seeds (Rahman et al., 2000; Sharma et al., 2004; Saboora and Kiarostami, 2006; Shila et al., 2016). Plant growth is ultimately reduced by salinity stress (Haque et al., 2008, 2014; Sikder et al., 2016). It is very important to know what extent of salinity a maize variety can tolerate at germination and early seedling growth stage. This information will help to identify salt tolerant varieties and/or genotypes and to develop saline soil management strategies. An experiment is therefore needed to investigate the effects of different levels of salinity on germination and early seedling growth of maize.

\section{Materials and methods}

The experiment was conducted at the laboratory of the Department of Soil Science, Patuakhali Science and Technology University, Dumki, Patuakhali during the period from January to February 2015.The experiment was designed at completely randomized design (CRD) with three replications. There were ten treatments consisting different levels of salinity. The treatments were $0,20,40,80,120,160,200,240,280$ and 320 $\mathrm{mM} \mathrm{NaCl}$ concentration. The germination experiment was conducted in petri dishes of $12 \mathrm{~cm}$ diameter lined with a thin layer of cotton. Bangladesh Agricultural Research Institute (BARI) developed "BARI Hybrid Maize 7" was used in the experiment. Twenty five seeds were placed on cotton bed in a circle pattern. Ten milli litre of treatment solutions of different salinity concentrations were poured in each Petri dish to immerse the seeds partially for ensuring proper aeration. Three replications were maintained for each salinity concentration. The petri dishes were placed on a table in the laboratory. The seeds were allowed to germinate at room temperature $\left(25 \pm 2^{\circ} \mathrm{C}\right)$. A $100 \mathrm{w}$ power bulb was fixed using a stand on the petri dishes. Required amount of distilled water was added to each petri dish every day to maintain same level of water as in initial date. Seeds were considered germinated when radicles measured $2 \mathrm{~mm}$ size.

The number of seeds germinated was recorded starting from 2 days after sowing (DAS) to 12 DAS. The results obtained in each day were converted into percentage. Shoot length was recorded at 7, 9, 11 and 13 DAS. Observation was made on germination percentage, fresh and dry weight of shoot and root, moisture content of root and shoot, seedling height, speed of germination and vigor index .Vigor index (VI) was worked out according to the formula.

$\mathrm{VI}=$ Germination $\% \times$ (Root length in $\mathrm{cm}+$ Shoot length in $\mathrm{cm}$ ) and

speed of germination was calculated as follows:

$s G=\frac{\text { Number of germinated seads }}{\text { Days of first ownt }}+\ldots+\frac{\text { Number of gaminated seeds }}{\text { Days of final cownt }}$

Data were statistically analyzed following F-test and the mean comparisons were made at 0.05 probability level by DMRT as outlined by Gomez and Gomez (1984).

\section{Results and Discussion}

Germination percentage: Germination percentage data was recorded starting from 3 DAS to 12 DAS. In 3 and 4 DAS some treatments had zero percent germination, thus statistical analysis was not performed in that days. In other days germination percentage was significantly influenced by different levels of salinity. Table 1 show that at 3 DAS 0 and $40 \mathrm{mM} \mathrm{NaCl}$ concentration had only 4 and $1.3 \%$ germination, respectively. After 1 day (at 4 DAS) more than $20 \%$ germination was found at $0,20,40$ and $80 \mathrm{mM} \mathrm{NaCl}$ concentration. At 5 DAS control treatment had $37.3 \%$ germination. The 20, 40, 80 and $120 \mathrm{mM}$ concentration had identical germination percentage both at $5(33.3 \%)$ and 6 DAS 
(41.3\%). With the passes of days the trend of germination was changed. All the days control treatment had highest germination and most of the days it was statistically similar with germination percentage obtained at $20 \mathrm{mM} \mathrm{NaCl}$ concentration. At all the sampling dates 20 and $40 \mathrm{mM}$ concentration had statistically similar germination percentage. More than $80 \%$ germination percentage was found only at 0,20 and $40 \mathrm{mM}$ concentration, which attained at 10, 11, and 12 DAS, respectively. Other concentrations had lower than $80 \%$ germination and were gradually decreased with the increase of concentration of salt in growth medium. The $80 \mathrm{mM} \mathrm{NaCl}$ concentration had $73.3 \%$ germination. Beyond this concentration the germination percentage was very poor. At 12 DAS 7.4 and $8.9 \%$ reduction in germination percentage was recorded at 20 and $40 \mathrm{mM} \mathrm{NaCl}$ concentration, respectively. The $80 \mathrm{mM} \mathrm{NaCl}$ concentration reduced germination percentage by $17.9 \%$. Thus upto $80 \mathrm{mM}$ $\mathrm{NaCl}$ concentration could be identified as acceptable for maize seed germination. Similar result was also found in rapeseed (Andalibi et al., 2005) and in sunflower (Shila et al., 2016). Ratnakara and Raib (2013) reported that though the lower concentrations of $\mathrm{NaCl}$ (upto $40 \mathrm{mM}$ ) did not affect percentage germination, the germination was found to be delayed in Triginella foenum-graecum. At higher salinity levels, inhibitory effect on germination was recorded to an extent that seeds did not germinate at $80 \mathrm{mM}$ and above concentrations of $\mathrm{NaCl}$ in Triginella foenumgraecum.

Table 1. Effects of different levels of salinity on germination percentage of maize (Zea mays L.)

\begin{tabular}{|c|c|c|c|c|c|c|c|c|c|c|c|}
\hline \multirow[t]{3}{*}{ Salt concentration } & \multicolumn{10}{|c|}{ Germination percentage } & \multirow{3}{*}{$\begin{array}{l}\text { Speed of } \\
\text { germination }\end{array}$} \\
\hline & 3 & 4 & 5 & 6 & 7 & 8 & 9 & 10 & 11 & 12 & \\
\hline & DAS & DAS & DAS & DAS & DAS & DAS & DAS & DAS & DAS & DAS & \\
\hline $\mathrm{T}_{1}: 0 \mathrm{mM} \mathrm{NaCl}$ & 4.0 & 28.0 & $37.3 a$ & $46.7 \mathrm{a}$ & $57.3 \mathrm{a}$ & $62.7 \mathrm{a}$ & $74.7 \mathrm{a}$ & $84.0 \mathrm{a}$ & $84.0 \mathrm{a}$ & $89.3 a$ & $20.9 a$ \\
\hline \multicolumn{12}{|l|}{ (Control) } \\
\hline $\mathrm{T}_{2}: 20 \mathrm{mM} \mathrm{NaCl}$ & 0.0 & 22.7 & $33.3 \mathrm{a}$ & $41.3 \mathrm{ab}$ & $46.7 b$ & $57.3 \mathrm{ab}$ & $62.7 \mathrm{~b}$ & $74.7 \mathrm{~b}$ & $80.0 \mathrm{a}$ & $82.7 \mathrm{ab}$ & $17.9 \mathrm{~b}$ \\
\hline $\mathrm{T}_{3}: 40 \mathrm{mM} \mathrm{NaCl}$ & 1.3 & 21.3 & $33.3 \mathrm{a}$ & $41.3 \mathrm{ab}$ & $46.7 b$ & $57.3 \mathrm{ab}$ & $61.3 \mathrm{~b}$ & $76.0 \mathrm{~b}$ & $78.7 \mathrm{a}$ & $81.3 b$ & $17.9 \mathrm{~b}$ \\
\hline $\mathrm{T}_{4}: 80 \mathrm{mM} \mathrm{NaCl}$ & 0.0 & 21.3 & $33.3 \mathrm{a}$ & 41.3ab & $45.3 b$ & $50.7 b$ & $58.7 \mathrm{~b}$ & $66.7 \mathrm{c}$ & $66.7 b$ & $73.3 \mathrm{c}$ & $16.6 \mathrm{~b}$ \\
\hline $\mathrm{T}_{5}: 120 \mathrm{mM} \mathrm{NaCl}$ & 0.0 & 18.7 & $33.3 \mathrm{a}$ & $41.3 \mathrm{ab}$ & $45.3 b$ & $50.7 b$ & $58.7 \mathrm{~b}$ & $64.0 \mathrm{c}$ & $65.3 b$ & $68.0 \mathrm{c}$ & $16.2 \mathrm{~b}$ \\
\hline $\mathrm{T}_{6}: 160 \mathrm{mM} \mathrm{NaCl}$ & 0.0 & 12.0 & $24.0 \mathrm{~b}$ & $34.7 b$ & $36.0 \mathrm{c}$ & $37.3 \mathrm{c}$ & $42.7 \mathrm{c}$ & $46.7 d$ & $48.0 \mathrm{c}$ & $54.7 \mathrm{~d}$ & $12.1 \mathrm{c}$ \\
\hline $\mathrm{T}_{7}: 200 \mathrm{mM} \mathrm{NaCl}$ & 0.0 & 4.0 & $9.3 \mathrm{c}$ & $16.0 \mathrm{c}$ & $21.3 \mathrm{~d}$ & $29.3 \mathrm{~cd}$ & $32.0 \mathrm{de}$ & $40.0 \mathrm{e}$ & $44.0 \mathrm{c}$ & $52.0 \mathrm{~d}$ & $8.0 \mathrm{~d}$ \\
\hline $\mathrm{T}_{8}: 240 \mathrm{mM} \mathrm{NaCl}$ & 0.0 & 1.3 & $13.3 \mathrm{c}$ & $18.7 \mathrm{c}$ & $22.7 d$ & $30.7 \mathrm{~cd}$ & $38.7 \mathrm{~cd}$ & $40.0 \mathrm{e}$ & $46.7 \mathrm{c}$ & $49.3 \mathrm{de}$ & $8.5 \mathrm{~d}$ \\
\hline $\mathrm{T}_{9}: 280 \mathrm{mM} \mathrm{NaCl}$ & 0.0 & 1.3 & $10.7 \mathrm{c}$ & $13.3 \mathrm{c}$ & $22.7 d$ & $32.0 \mathrm{~cd}$ & $34.7 \mathrm{~cd}$ & $38.7 \mathrm{e}$ & $41.3 \mathrm{~cd}$ & 42.7ef & $7.7 \mathrm{de}$ \\
\hline $\mathrm{T}_{10}: 320 \mathrm{mM} \mathrm{NaCl}$ & 0.0 & 0.0 & $4.0 \mathrm{~d}$ & $12.0 \mathrm{c}$ & $17.3 \mathrm{~d}$ & $24.0 \mathrm{~d}$ & $25.3 \mathrm{e}$ & $30.7 f$ & $34.7 \mathrm{~d}$ & $38.7 f$ & $5.8 \mathrm{e}$ \\
\hline$\% \mathrm{CV}$ & & & 12.9 & 14.09 & 12.13 & 12.31 & 10.34 & 6.24 & 8.59 & 7.89 & 8.66 \\
\hline Significance level & & & $* * *$ & $* * *$ & $* * *$ & $* * *$ & $* * *$ & $* * *$ & $* * *$ & $* * *$ & $* * *$ \\
\hline $\mathrm{SE}( \pm)$ & & & 1.738 & 2.494 & 2.530 & 3.070 & 2.921 & 2.022 & 2.921 & 2.860 & 0.659 \\
\hline
\end{tabular}

Common letters in a column are not significantly different at 5\% level by DMRT

Speed of germination: Speed of germination was significantly influenced by different levels of salinity. Speed of germination ranged from 5.8 to 20.9 , highest being in control and lowest in $320 \mathrm{mM} \mathrm{NaCl}$ concentration (Table1). The second highest position was ranked by the $20 \mathrm{mM} \mathrm{NaCl}$ concentration which was statistically similar with 40,80 and $120 \mathrm{mM} \mathrm{NaCl}$ concentration. Due to use of increasing rate of salt concentration, speed of germination reduced by 14.15 to $72.16 \%$. 
Seedling height: Seedling height data was recorded at $7,9,11,13$ and 15 days after sowing (DAS). In all the data recording day seedling height was significantly influenced by different levels of salinity. At 7 DAS highest seedling height was found in control treatment $(2.17 \mathrm{~cm})$ and it was statistically similar with that obtained at $20 \mathrm{mM} \mathrm{NaCl}(2.04 \mathrm{~cm})$. With the increase of the concentration of solution seedling height was gradually decreased (Table 2). At 9 DAS the seedling height pattern was slightly changed. This day highest height was further found at control treatment and it was statistically similar with 20 and $40 \mathrm{mM} \mathrm{NaCl}$ concentration. In other concentrations, seedling height gradually decreased with increasing concentration of solution. At 11 DAS highest seedling height was found at control treatment $(10.81 \mathrm{~cm})$ and it was statistically similar with 20 (10.47), $40(10.39 \mathrm{~cm})$ and $80(10.32$ $\mathrm{cm}) \mathrm{mM} \mathrm{NaCl}$ concentration. At 13 DAS similar trend was observed. The result clearly indicates that up to 80 $\mathrm{mM} \mathrm{NaCl}$ concentration, salinity is not harmful for maize seedling height. However, at 20, 40 and $80 \mathrm{mM}$ $\mathrm{NaCl}$ concentration had better performance compared to control. The results further showed that with the increase of concentration of salt, plant takes longer time for their growth and development15 DAS.

Shoot elongation rate: Shoot elongation rate was calculated at 7, 9, 11, 13 and 15 DAS, and was significantly influenced by salinity. At 7, 9, 11, 13 and 15 DAS shoot elongation rate ranged from 0.13 to $3.10,0.96$ to $7.78,1.10$ to $9.83,1.26$ to 11.10 and 1.89 to $13.58 \mathrm{~mm} /$ day, respectively (Table 3 ).

Table 2. Effects of different levels of salinity on seedling height of maize

\begin{tabular}{|c|c|c|c|c|c|c|c|c|c|c|}
\hline \multirow[t]{2}{*}{ Salt concentration } & \multicolumn{2}{|c|}{7 DAS } & \multicolumn{2}{|r|}{9 DAS } & \multicolumn{2}{|r|}{11 DAS } & \multicolumn{2}{|c|}{13 DAS } & \multicolumn{2}{|c|}{$15 \mathrm{DAS}$} \\
\hline & $\begin{array}{c}\text { Seedling } \\
\text { height } \\
(\mathrm{cm})\end{array}$ & $\begin{array}{c}\% \% \\
\text { decrease } \\
\text { over } \\
\text { control }\end{array}$ & $\begin{array}{c}\text { Seedling } \\
\text { height } \\
(\mathrm{cm})\end{array}$ & $\begin{array}{c}\% \% \\
\text { decrease } \\
\text { over } \\
\text { control }\end{array}$ & $\begin{array}{c}\text { Seedling } \\
\text { height } \\
(\mathrm{cm})\end{array}$ & $\begin{array}{c}\% \\
\text { decrease } \\
\text { over } \\
\text { control }\end{array}$ & $\begin{array}{c}\text { Seedling } \\
\text { height } \\
(\mathrm{cm})\end{array}$ & $\begin{array}{c}\% \% \\
\text { decrease } \\
\text { over } \\
\text { control }\end{array}$ & $\begin{array}{c}\text { Seedling } \\
\text { height } \\
(\mathrm{cm})\end{array}$ & $\begin{array}{c}\% \\
\text { decrease } \\
\text { over } \\
\text { control }\end{array}$ \\
\hline $\begin{array}{l}\mathrm{T}_{1}: 0 \mathrm{mM} \mathrm{NaCl} \\
\text { (Control) }\end{array}$ & $2.17 \mathrm{a}$ & - & $7.00 \mathrm{a}$ & - & $10.81 \mathrm{a}$ & - & $14.43 \mathrm{a}$ & - & $20.16 \mathrm{a}$ & - \\
\hline $\mathrm{T}_{2}: 20 \mathrm{mM} \mathrm{NaCl}$ & $2.04 \mathrm{ab}$ & 5.99 & $6.87 \mathrm{a}$ & 1.90 & $10.47 \mathrm{a}$ & 3.11 & $14.08 \mathrm{a}$ & 2.43 & $20.37 \mathrm{a}$ & -1.03 \\
\hline $\mathrm{T}_{3}: 40 \mathrm{mM} \mathrm{NaCl}$ & $1.94 \mathrm{~b}$ & 10.75 & $6.84 \mathrm{a}$ & 2.29 & $10.39 \mathrm{a}$ & 3.85 & $14.19 \mathrm{a}$ & 1.69 & $21.51 \mathrm{a}$ & -6.71 \\
\hline $\mathrm{T}_{4}: 80 \mathrm{mM} \mathrm{NaCl}$ & $1.71 \mathrm{c}$ & 21.04 & $6.33 b$ & 9.52 & $10.32 \mathrm{a}$ & 4.53 & $14.07 \mathrm{a}$ & 2.47 & $20.50 \mathrm{a}$ & -1.69 \\
\hline $\mathrm{T}_{5}: 120 \mathrm{mM} \mathrm{NaCl}$ & $1.31 \mathrm{~d}$ & 39.78 & $5.37 \mathrm{c}$ & 23.33 & $8.84 b$ & 18.22 & $11.97 \mathrm{~b}$ & 17.07 & $18.05 b$ & 10.48 \\
\hline $\mathrm{T}_{6}: 160 \mathrm{mM} \mathrm{NaCl}$ & $1.09 \mathrm{e}$ & 49.92 & $3.93 \mathrm{~d}$ & 43.90 & $6.93 \mathrm{c}$ & 35.86 & $9.49 \mathrm{c}$ & 34.26 & $13.31 \mathrm{c}$ & 33.99 \\
\hline $\mathrm{T}_{7}: 200 \mathrm{mM} \mathrm{NaCl}$ & $0.34 \mathrm{f}$ & 84.49 & $1.49 \mathrm{e}$ & 78.67 & $3.23 \mathrm{~d}$ & 70.15 & $5.13 \mathrm{~d}$ & 64.43 & $7.79 \mathrm{~d}$ & 61.34 \\
\hline $\mathrm{T}_{8}: 240 \mathrm{mM} \mathrm{NaCl}$ & $0.22 \mathrm{fg}$ & 89.86 & $1.19 \mathrm{ef}$ & 82.95 & $1.86 \mathrm{e}$ & 82.82 & $2.67 \mathrm{e}$ & 81.47 & $5.28 \mathrm{e}$ & 73.81 \\
\hline $\mathrm{T}_{9}: 280 \mathrm{mM} \mathrm{NaCl}$ & $0.17 \mathrm{fg}$ & 92.32 & $1.14 \mathrm{ef}$ & 83.71 & $1.77 \mathrm{ef}$ & 83.66 & $2.71 \mathrm{e}$ & 81.20 & $4.72 \mathrm{ef}$ & 76.59 \\
\hline $\mathrm{T}_{10}: 320 \mathrm{mM} \mathrm{NaCl}$ & $0.09 \mathrm{~g}$ & 95.70 & $0.86 \mathrm{f}$ & 87.71 & $1.21 \mathrm{f}$ & 88.78 & $1.63 \mathrm{e}$ & 88.68 & $2.84 \mathrm{f}$ & 85.93 \\
\hline$\% \mathrm{CV}$ & 11.51 & & 6.84 & & 4.94 & & 7.15 & & 8.83 & \\
\hline Significance level & $* * *$ & & $* * *$ & & $* * *$ & & $* * *$ & & $* * *$ & \\
\hline $\operatorname{SE}( \pm)$ & 0.0730 & & 01623 & & 0.1680 & & 0.3733 & & 0.6856 & \\
\hline
\end{tabular}

Common letters in a column are not significantly different at $5 \%$ level by DMRT

At 7 DAS highest elongation rate was found at control treatment. In this date second $(2.91 \mathrm{~mm} /$ day $)$ and third position $(2.77 \mathrm{~mm} /$ day $)$ was ranked by 20 and $40 \mathrm{mM}$ $\mathrm{NaCl}$ concentration. At 9 DAS highest position was further ranked at control treatment, second (7.63 $\mathrm{mm} /$ day) and third position was recorded by 20 and 40 $\mathrm{mM} \mathrm{NaCl}$ concentration, respectively. At 11 DAS highest position was ranked at control treatment, second $(9.52 \mathrm{~mm} /$ day $)$ and third position was recorded by 20 and $40 \mathrm{mM} \mathrm{NaCl}$ concentration, respectively. At 
13 DAS highest position was ranked at control treatment, second (10.91 $\mathrm{mm} /$ day) and third position was recorded by 40 and $20 \mathrm{mM} \mathrm{NaCl}$ concentration, respectively. At $15 \mathrm{DAS}$ the highest position was ranked by $40 \mathrm{mM} \mathrm{NaCl}$ concentration, the $20 \mathrm{mM}$ $\mathrm{NaCl}$ concentration and control had the second and third position, respectively. Table 3 further shows that increasing salt concentration reduced the shoot elongation rate at every sampling date. Elongation rate in different salt concentrations also varied in different sampling dates. At lower concentration higher elongation rate was found at initial dates but it was gradually delayed with the increase of salt concentration. Shila et al. (2016) also noticed similar results in sunflower.

Table 3. Effects of different levels of salinity on shoot elongation rate of maize (Zea mays L.)

\begin{tabular}{|c|c|c|c|c|c|c|c|c|c|c|}
\hline \multirow{2}{*}{$\begin{array}{c}\text { Salt } \\
\text { concentration }\end{array}$} & \multicolumn{2}{|c|}{$7 \mathrm{DAS}$} & \multicolumn{2}{|c|}{9 DAS } & \multicolumn{2}{|c|}{$11 \mathrm{DAS}$} & \multicolumn{2}{|c|}{13 DAS } & \multicolumn{2}{|c|}{$15 \mathrm{DAS}$} \\
\hline & $\begin{array}{c}\text { Shoot } \\
\text { elongation } \\
\text { rate }\end{array}$ & $\begin{array}{c}\% \\
\text { decrease } \\
\text { over } \\
\text { control }\end{array}$ & $\begin{array}{l}\text { Seedling } \\
\text { height } \\
(\mathrm{cm})\end{array}$ & $\begin{array}{c}\% \\
\text { decrease } \\
\text { over } \\
\text { control }\end{array}$ & $\begin{array}{l}\text { Seedling } \\
\text { height } \\
(\mathrm{cm})\end{array}$ & $\begin{array}{c}\% \% \\
\text { decrease } \\
\text { over } \\
\text { control }\end{array}$ & $\begin{array}{l}\text { Seedling } \\
\text { height } \\
(\mathrm{cm})\end{array}$ & $\begin{array}{c}\% \% \\
\text { decrease } \\
\text { over } \\
\text { control }\end{array}$ & $\begin{array}{l}\text { Seedling } \\
\text { height } \\
(\mathrm{cm})\end{array}$ & $\begin{array}{c}\% \\
\text { decrease } \\
\text { over } \\
\text { control }\end{array}$ \\
\hline $\begin{array}{l}\mathrm{T}_{1}: 0 \mathrm{mM} \mathrm{NaCl} \\
\text { (Control) }\end{array}$ & $3.10 \mathrm{a}$ & & $7.78 \mathrm{a}$ & & $9.83 a$ & & $11.10 \mathrm{a}$ & & $13.44 \mathrm{a}$ & \\
\hline $\begin{array}{l}\mathrm{T}_{2}: 20 \mathrm{mM} \\
\mathrm{NaCl}\end{array}$ & $2.91 \mathrm{ab}$ & 5.99 & $7.63 \mathrm{a}$ & 1.93 & $9.52 \mathrm{a}$ & 3.14 & $10.83 \mathrm{a}$ & 2.43 & $13.58 \mathrm{a}$ & -1.03 \\
\hline $\begin{array}{l}\mathrm{T}_{3}: 40 \mathrm{mM} \\
\mathrm{NaCl}\end{array}$ & $2.77 b$ & 10.75 & $7.60 \mathrm{a}$ & 2.31 & $9.45 \mathrm{a}$ & 3.88 & $10.91 \mathrm{a}$ & 1.69 & $14.34 \mathrm{a}$ & -6.71 \\
\hline $\begin{array}{l}\mathrm{T}_{4}: 80 \mathrm{mM} \\
\mathrm{NaCl}\end{array}$ & $2.45 \mathrm{c}$ & 21.04 & $7.04 \mathrm{~b}$ & 9.55 & $9.38 \mathrm{a}$ & 4.56 & $10.83 a$ & 2.47 & $13.67 \mathrm{a}$ & -1.69 \\
\hline $\begin{array}{l}\mathrm{T}_{5}: 120 \mathrm{mM} \\
\mathrm{NaCl}\end{array}$ & $1.87 \mathrm{~d}$ & 39.78 & $5.96 \mathrm{c}$ & 23.36 & $8.04 b$ & 18.25 & $9.21 \mathrm{~b}$ & 17.07 & $12.03 \mathrm{~b}$ & 10.48 \\
\hline $\begin{array}{l}\mathrm{T}_{6}: 160 \mathrm{mM} \\
\mathrm{NaCl}\end{array}$ & $1.55 \mathrm{e}$ & 49.92 & $4.36 \mathrm{~d}$ & 43.92 & $6.30 \mathrm{c}$ & 35.88 & $7.30 \mathrm{c}$ & 34.26 & $8.87 \mathrm{c}$ & 33.99 \\
\hline $\begin{array}{l}\mathrm{T}_{7}: 200 \mathrm{mM} \\
\mathrm{NaCl}\end{array}$ & $0.48 \mathrm{f}$ & 84.49 & $1.66 \mathrm{e}$ & 78.67 & $2.93 \mathrm{~d}$ & 70.16 & $3.95 \mathrm{~d}$ & 64.43 & $5.20 \mathrm{~d}$ & 61.34 \\
\hline $\begin{array}{l}\mathrm{T}_{8}: 240 \mathrm{mM} \\
\mathrm{NaCl}\end{array}$ & $0.31 \mathrm{fg}$ & 89.86 & $1.33 \mathrm{ef}$ & 82.96 & $1.69 \mathrm{e}$ & 82.83 & $2.06 \mathrm{e}$ & 81.47 & $3.52 \mathrm{e}$ & 73.81 \\
\hline $\begin{array}{l}\mathrm{T}_{9}: 280 \mathrm{mM} \\
\mathrm{NaCl}\end{array}$ & $0.24 \mathrm{fg}$ & 92.32 & $1.27 \mathrm{ef}$ & 83.72 & $1.61 \mathrm{ef}$ & 83.66 & $2.09 \mathrm{e}$ & 81.20 & $3.15 \mathrm{ef}$ & 76.59 \\
\hline $\begin{array}{l}\mathrm{T}_{10}: 320 \mathrm{mM} \\
\mathrm{NaCl}\end{array}$ & $0.13 \mathrm{~g}$ & 95.70 & $0.96 \mathrm{f}$ & 87.72 & $1.10 \mathrm{f}$ & 88.78 & $1.26 \mathrm{e}$ & 88.68 & $1.89 \mathrm{f}$ & 85.93 \\
\hline$\% \mathrm{CV}$ & 11.45 & & 6.89 & & 4.97 & & 7.13 & & 8.83 & \\
\hline $\begin{array}{l}\text { Significance } \\
\text { level }\end{array}$ & $* * *$ & & $* * *$ & & $* * *$ & & $* * *$ & & $* * *$ & \\
\hline $\mathrm{SE}( \pm)$ & 0.1049 & & 0.1817 & & 0.1713 & & 0.2858 & & 0.4572 & \\
\hline
\end{tabular}

Common letters in a column are not significantly different at $5 \%$ level by DMRT

Root length: Ten different levels of salinity had significant effect on root length of maize. The root length ranged from $2.85 \mathrm{~cm}$ to $23.61 \mathrm{~cm}$ (Table 4). The highest root length $(23.61 \mathrm{~cm})$ was found in $20 \mathrm{mM}$ $\mathrm{NaCl}$ concentration which was $5.6 \%$ higher than the control treatment; although it was statistically similar with control treatment $(22.36 \mathrm{~cm})$. Beyond $20 \mathrm{mM}$ $\mathrm{NaCl}$ concentration the root length was found reduced gradually and ultimately in the highest concentration root length was the lowest. In the experiment $12.3 \%$ root length reduction was found in $80 \mathrm{mM} \mathrm{NaCl}$ concentration, beyond this level root length was reduced drastically and in $320 \mathrm{mM} \mathrm{NaCl}$ concentration 
root length reduced by about $87 \%$. In fact in this concentration roots were lean and thin.

Root fresh weight: There was a significant effect of salinity on root fresh weight of maize. Root fresh weight was ranged from 0.415 to $4.168 \mathrm{~g}$ over different levels of salinity (Table 4). Highest of $4.168 \mathrm{~g}$ fresh root was found at $0 \mathrm{mM} \mathrm{NaCl}$ concentration. Second highest root weight was found in $20 \mathrm{mM} \mathrm{NaCl}$ concentration. There was found a decreasing trend of root fresh weight, with the increasing of the concentration of the solution. In the experiment $20 \mathrm{mM}$ $\mathrm{NaCl}$ concentration had $7.88 \%$ decrease over control, beyond this concentration a drastic reduction was observed. At $240 \mathrm{mM} \mathrm{NCl}$ concentration more than $80 \%$ reduction in root fresh weight was observed. At $320 \mathrm{mM} \mathrm{NaCl}$ concentration root fresh weight reduced by $90.1 \%$.

Root dry weight: Root dry weight was significantly influenced by different levels of salinity. Root dry weight ranged from $0.038 \mathrm{~g}$ in $320 \mathrm{mM} \mathrm{NaCl}$ concentration to $0.403 \mathrm{~g}$ in $0 \mathrm{mM} \mathrm{NaCl}$ concentration (control) (Table 4). Second highest (0.360 g) result was obtained in $20 \mathrm{mM} \mathrm{NaCl}$ concentration which had 10.6 $\%$ reduction over control. In the experiment $40 \mathrm{mM}$ $\mathrm{NaCl}$ concentration had less than $20 \%$ reduction in root dry weight. However, with the increase of salt concentration root dry weight was drastically reduced. About $90.6 \%$ reduction in root dry weight was observed due to use of $320 \mathrm{mM} \mathrm{NaCl}$ concentration.

Shoot fresh weight: Different levels of salinity had significant effect on shoot fresh weight of maize. Shoot fresh weight ranged from $0.661 \mathrm{~g}$ in $320 \mathrm{mM} \mathrm{NaCl}$ concentration to $5.478 \mathrm{~g}$ in $20 \mathrm{mM} \mathrm{NaCl}$ concentration (Table 4). Highest of $5.478 \mathrm{~g}$ shoot fresh weight in 20 $\mathrm{mM} \mathrm{NaCl}$ concentration was statistically similar with 0,40 and $80 \mathrm{mM} \mathrm{NaCl}$ concentration. Beyond $20 \mathrm{mM}$ $\mathrm{NaCl}$ concentration, with the increase of the concentration of the solution shoot fresh weight was reduced gradually. Compared to control treatment 20 $\mathrm{mM} \mathrm{NaCl}$ concentration gave $0.55 \%$ higher fresh weight and upto $80 \mathrm{mM} \mathrm{NaCl}$ concentration shoot fresh weight reduced by only $6.9 \%$. After this concentration the reduction rate increased very fast. In the experiment $320 \mathrm{mM} \mathrm{NaCl}$ concentration had 87.9 $\%$ reduction in shoot fresh weight. Thus upto $80 \mathrm{mM}$ $\mathrm{NaCl}$ concentration was found acceptable for production of shoot fresh weight.

Shoot dry weight: Shoot dry weight was significantly influenced by different levels of salinity. The lowest dry weight of $0.092 \mathrm{~g}$ was found in $320 \mathrm{mM} \mathrm{NaCl}$ concentration and was highest of $0.515 \mathrm{~g}$ in $0 \mathrm{mM}$ $\mathrm{NaCl}$ concentration ( Table 4) .In the experiment 0,20 , 40, 80 and $120 \mathrm{mM} \mathrm{NaCl}$ concentration gave statistically similar shoot dry weight. Although not significant but $20,40,80$, and $120 \mathrm{mM} \mathrm{NaCl}$ concentration gave $1.42,0.84,5.95$ and $9.58 \%$, respectively lower shoot dry weight than control treatment. The result announces that upto $120 \mathrm{mM}$ $\mathrm{NaCl}$ concentration maize can tolerate salinity. In the experiment $320 \mathrm{mM} \mathrm{NaCl}$ concentration had highest of $82 \%$ reduction.

Shoot and root moisture content: Shoot moisture content was significantly influenced by different levels of salinity. Table 4 shows that highest of $90.7 \%$ moisture content was recorded by the treatment $20 \mathrm{mM}$ $\mathrm{NaCl}$, however, it was statistically similar to the treatment 0,40 and $80 \mathrm{mM} \mathrm{NaCl}$ concentration. Beyond $80 \mathrm{mM} \mathrm{NaCl}$ concentration increasing salt concentration reduced shoot moisture content. In other word, increasing salt concentration increase dry matter accumulation by reducing water uptake by the plant. Root moisture content was less affected by salinity compared to shoot moisture content. It was also significantly influenced by different levels of salinity. Root moisture content varied between 90.7 to $85.7 \%$ at salt concentration of $0-320 \mathrm{mM} \mathrm{NaCl}$ concentration. It indicates that under this range salinity has no effect on root moisture content.

Vigor index: Salinity effect on vigor index was significant. Vigor index value ranged from 2.2 to 38.1 over the different concentration of solution (Table 4). The control had highest vigor index (38.1). Second 
highest value (36.4) was recorded at $20 \mathrm{mM} \mathrm{NaCl}$ concentration. Increasing concentration of salt gradually decrease the vigor index. When vigor index value were compared with control treatment it was found that the 20 and $40 \mathrm{mM} \mathrm{NaCl}$ concentration had 4.53 and $9.98 \%$ decrease, rspectively. As high as $94.2 \%$ decrease in vigor index was found at highest concentration of $320 \mathrm{mM} \mathrm{NaCl}$.

Table 4. Effects of different levels of salinity on different growth parameters of maize

\begin{tabular}{|c|c|c|c|c|c|c|c|c|}
\hline Salt concentration & $\begin{array}{c}\text { Root } \\
\text { length }(\mathrm{cm})\end{array}$ & $\begin{array}{r}\text { Root } \\
\text { fresh } \\
\text { weight }\end{array}$ & $\begin{array}{c}\text { Root } \\
\text { dry } \\
\text { weight }\end{array}$ & $\begin{array}{c}\text { Shoot fresh } \\
\text { weight }\end{array}$ & $\begin{array}{c}\text { Shoot dry } \\
\text { weight }\end{array}$ & $\begin{array}{c}\text { Shoot } \\
\text { moisture } \\
\text { content }(\%)\end{array}$ & $\begin{array}{c}\text { Root } \\
\text { moisture } \\
\text { content }(\%)\end{array}$ & $\begin{array}{l}\text { Vigor } \\
\text { index }\end{array}$ \\
\hline $\begin{array}{l}\mathrm{T}_{1}: 0 \mathrm{mM} \mathrm{NaCl} \\
\text { (Control) }\end{array}$ & $22.36 \mathrm{ab}$ & $4.168 \mathrm{a}$ & $0.403 a$ & $5.448 \mathrm{a}$ & $0.515 \mathrm{a}$ & $90.5 \mathrm{a}$ & $90.3 \mathrm{ab}$ & $38.1 \mathrm{a}$ \\
\hline $\mathrm{T}_{2}: 20 \mathrm{mM} \mathrm{NaCl}$ & $23.61 \mathrm{a}$ & $3.840 \mathrm{~b}$ & $0.360 \mathrm{~b}$ & $5.478 \mathrm{a}$ & $0.508 \mathrm{a}$ & $90.7 \mathrm{a}$ & $90.6 \mathrm{a}$ & $36.4 \mathrm{a}$ \\
\hline $\mathrm{T}_{3}: 40 \mathrm{mM} \mathrm{NaCl}$ & $20.75 b c$ & $3.263 \mathrm{c}$ & $0.330 \mathrm{c}$ & $5.369 \mathrm{a}$ & $0.511 \mathrm{a}$ & $90.5 \mathrm{a}$ & $89.9 \mathrm{ab}$ & $34.3 \mathrm{a}$ \\
\hline $\mathrm{T}_{4}: 80 \mathrm{mM} \mathrm{NaCl}$ & $19.60 \mathrm{c}$ & $2.978 \mathrm{~d}$ & $0.309 \mathrm{~d}$ & $5.074 \mathrm{a}$ & $0.484 \mathrm{a}$ & $90.4 \mathrm{a}$ & $89.6 \mathrm{ab}$ & $29.4 b$ \\
\hline $\mathrm{T}_{5}: 120 \mathrm{mM} \mathrm{NaCl}$ & $16.06 \mathrm{~d}$ & $2.600 \mathrm{e}$ & $0.277 \mathrm{e}$ & $4.408 b$ & $0.466 \mathrm{a}$ & $89.4 \mathrm{a}$ & $89.3 \mathrm{ab}$ & $23.2 \mathrm{c}$ \\
\hline $\mathrm{T}_{6}: 160 \mathrm{mM} \mathrm{NaCl}$ & $11.82 \mathrm{e}$ & $2.147 f$ & $0.232 \mathrm{f}$ & $3.468 \mathrm{c}$ & $0.343 b$ & $90.1 \mathrm{a}$ & $89.2 \mathrm{ab}$ & $13.7 \mathrm{~d}$ \\
\hline $\mathrm{T}_{7}: 200 \mathrm{mM} \mathrm{NaCl}$ & $7.74 \mathrm{f}$ & $1.184 \mathrm{~g}$ & $0.143 \mathrm{~g}$ & $1.537 \mathrm{~d}$ & $0.191 \mathrm{c}$ & $87.6 \mathrm{~b}$ & $87.7 \mathrm{bc}$ & $8.0 \mathrm{e}$ \\
\hline $\mathrm{T}_{8}: 240 \mathrm{mM} \mathrm{NaCl}$ & $4.75 \mathrm{~g}$ & $0.821 \mathrm{~h}$ & $0.124 \mathrm{~h}$ & $0.889 \mathrm{e}$ & $0.177 \mathrm{c}$ & $80.1 \mathrm{~d}$ & $84.9 \mathrm{~d}$ & $4.9 \mathrm{ef}$ \\
\hline $\mathrm{T}_{9}: 280 \mathrm{mM} \mathrm{NaCl}$ & $4.63 \mathrm{~g}$ & $0.777 \mathrm{~h}$ & $0.111 \mathrm{i}$ & $0.804 \mathrm{e}$ & $0.123 d$ & $84.5 \mathrm{c}$ & $85.7 \mathrm{~cd}$ & $4.0 \mathrm{f}$ \\
\hline $\mathrm{T}_{10}: 320 \mathrm{mM} \mathrm{NaCl}$ & $2.85 \mathrm{~g}$ & $0.415 \mathrm{i}$ & $0.038 \mathrm{j}$ & $0.661 \mathrm{e}$ & $0.092 \mathrm{~d}$ & $86.1 \mathrm{bc}$ & $90.7 \mathrm{a}$ & $2.2 \mathrm{f}$ \\
\hline$\% \mathrm{CV}$ & 10.40 & 7.60 & 9.12 & 9.72 & 6.04 & 1.19 & 1.62 & 11.44 \\
\hline Significance level & $* * *$ & $* * *$ & $* * *$ & $* * *$ & $* * *$ & $* * *$ & $* * *$ & $* * *$ \\
\hline $\operatorname{SE}( \pm)$ & 0.8056 & 0.0966 & 0.0002 & 0.1862 & 0.018 & 0.6069 & 0.8307 & 1.283 \\
\hline
\end{tabular}

Common letters in a column are not significantly different at $5 \%$ level by DMR

\section{Conclusion}

Salinity reduced germination percentage of maize and the extent of reduction increased with the increase in the concentration of salinity in the growth medium. $\mathrm{NaCl}$ concentration up to $80 \mathrm{mM}$ was found as safe for maize seed germination. $\mathrm{NaCl}$ upto $40 \mathrm{mM}$ favors plant growth which otherwise indicates the necessity of $\mathrm{NaCl}$ in lower concentration for plant growth and development. At higher concentration like $320 \mathrm{mM}$ $\mathrm{NaCl}$, root was more affected than shoot. Further study is needed to test the performance of different maize genotypes at varying degree of salt stress.

\section{Acknowledgement}

The author great fully acknowledges financial support provided by Bangladesh Agricultural Research Council (BARC) under the project entitled "Increasing fertilizer and irrigation water use efficiency for crop production in southern coastal saline soils of Bangladesh" to conduct the study.

\section{References}

Andalibi B, Zangani E, Nazari AH (2005). Effects of water stress on germination indices in six rapeseed cultivars (Brassica napus L.). Iranian Journal of Agricultural Sciences 36: 457-463.

Carpıcı EB, Celık N, Bayram G (2009). Effects of salt stress on germination of some maize (Zea mays L.) cultivars. African Journal of Biotechnology, 8(19): 4918-4922.

Foolad MR, Hyman JR, Lin GY (1999). Relationships between Cold and Salt Tolerance During Seed Germination in Tomato: Analysis of Response and Correlated Response to Selection. Plant Breeding, 118: 49-52. 
Foolad MR, Lin GY (1997). Genetic Potential for Salt Tolerance during Germination in Lycopersicon Species. Horticultural Science, 32: 296-300.

Gomez KA, Gomez AA (1984). Statistical Procedures for Agricultural Research, John Wiley and Sons, New York.

Haque MA, Jahiruddin M, Hoque MA, Rahman MZ, Clarke D (2014). Temporal variability of soil and water salinity and its effect on crop at kalapara upazila. Journal of Environmental Science \& Natural Resources, 7(2): 111-114.

Haque MA, Jharna DE, Hoque MF, Uddin MN, Saleque MA (2008). Soil solution electrical conductivity and basic cations composition in the rhizosphere of lowland rice in coastal soils. Bangladesh Journal of Agrcultural Research, 33(2): 243-250.

Rahman M, Kayani SA, Gul S (2000). Combined Effects of Temperature and Salinity Stress on Corn Sunahry Cv., Pak. J. Biological Sci., 3(9): 1459-1463.

Ratnakara A, Raib A (2013). Effect of sodium chloride salinity on seed germination and early seedling growth of trigonella Foenum-graecum L. Journal of Environmental Research, 1(4): 304-309.
Roychoudhury A, Chakraborty M (2013). Biochemical and molecular basis of varietal difference in plant salt tolerance. Annual Review \& Research in Biology, 3(4): 422-454.

Saboora A, Kiarostami K (2006). Salinity $(\mathrm{NaCl})$ Tolerance of Wheat Genotypes at Germination and Early Seedling Growth. Pak. J.Biological Sci., 9(11): 2009-2021.

Sharma AD, Thakur M, Rana M, Singh K (2004) Effect of Plant Growth Hormones and Abiotic Stresses on Germination, Growth and Phosphatase Activities in Sorghum bicolor (L.) Moench Seeds. Afr. J. Biotechnol., 3: 308-312.

Shila A, Haque MA, Ahmed R, Howlader MHK (2016). Effect of different levels of salinity on germination and early seedling growth of sunflower. World Research Journal of Agricultural Sciences, 3(1): 048-053.

Sikder MU, Haque MA, Jodder R, Kumar T, Mondal D (2016). Polythene mulch and irrigation for mitigation of salinity effects on maize (Zea mays L.) The Agriculturists, 14(2): 01-13.

SRDI (2010). SRMAF Project. Soil Resource Development Institute. Ministry of Agriculture GoB. 